\title{
Conductivities and Ion Association of Quaternary Ammonium Carboxylates in $\gamma$-Butyrolactone
}

\author{
Makoto UE*, Tomohiro SATO and Masayuki TAKEDA \\ Received January 25, 1993 ; Accepted February 23, 1993
}

\begin{abstract}
The conductivities for eighteen quaternary ammonium carboxylates in $\gamma$-butyrolactone have been measured at $25^{\circ} \mathrm{C}$, which have been recently used as high performance liquid electrolytes in aluminum electrolytic capacitors. The limiting molar conductivities and the ion association constants of these electrolytes were calculated by the Shedlovsky extrapolation method. The single ion limiting molar conductivities of carboxylate ions were $24.8,20.2$ and $18.9 \mathrm{~S} \mathrm{~cm}^{2} \mathrm{~mol}^{-1}$ for bimaleate, biphthalate and benzoate ion, respectively. The single ion limiting molar conductivities of quaternary ammonium ions decreased in the order: $\mathrm{Me}_{4} \mathrm{~N}^{+} 23.1>\mathrm{Me}_{3} \mathrm{EtN}^{+} 22.7>\mathrm{Me}_{2}\left(\mathrm{CH}_{2}\right)_{4} \mathrm{~N}^{+} 22.3>$ $\mathrm{Me}_{2} \mathrm{Et}_{2} \mathrm{~N}^{+} 22.0>\mathrm{MeEt}_{3} \mathrm{~N}^{+} 21.3>\mathrm{Et}_{4} \mathrm{~N}^{+} 20.6 \mathrm{~S} \mathrm{~cm}^{2} \mathrm{~mol}^{-1}$. The ion association constants increased as the ionic radius of a quaternary ammnonium ion and the acidity of a carboxylic acid decreased.
\end{abstract}

\section{INTRODUCTION}

The conductometric analysis of nonaqueous electrolytes ${ }^{1-3)}$ containing $\gamma$-butyrolactone (GBL) solvent has been focused on tetraalkylammnonium and alkali metal salts of strong acids ${ }^{4,5}$, particularly on the lithium salts ${ }^{6-8)}$ such as $\mathrm{LiClO}_{4}, \mathrm{LiBF}_{4}, \mathrm{LiPF}_{6}$ and $\mathrm{LiAsF}_{6}$, because $\gamma$-butyrolactone is an important solvent used for high energy lithium batteries.

On the other hand, quaternary ammonium salts of carboxylic acids in $\gamma$-butyrolactone have recently acquired a potential application in aluminum electrolytic capacitors ${ }^{9-11)}$. However, the conductivity data for quaternary ammonium carboxylates have been reported only for acetonitrile $(\mathrm{AN})^{12-14)}, \mathrm{N}, \mathrm{N}-$ dimethylformamide (DMF) ${ }^{15-17)}$ and propylene carbonate (PC) ${ }^{18)}$.

This work is a systematic study on the transport properties of quaternary ammonium carboxylate/ $\gamma$ butyrolactone electrolytes, which are used in aluminum

Tsukuba Research Centre, Mitsubishi Petrochemical Co., Ltd. (8-3-1 Chuo, Ami, Inashiki, Ibaraki 300-03, Japan)

Key words: Conductivity, Association constant, Quatemary ammonium carboxylate, $\gamma$-Butyrolactone electrolytic capacitors. The conductivity data of several quaternary ammonium bimaleates, biphthalates and benzoates, which involve symmetric and asymmetric cations from tetramethylammonium to tetraethylammonium, are presented ${ }^{19}$.

\section{EXPERIMENTAL}

Tetraethylammonium bimaleate, biphthalate and benzoate were prepared by the neutralization of maleic acid (Tokyo Chemical Industry Co.), phthalic acid (Tokyo Chemical Industry Co.) and benzoic acid (Wako Pure Chemical Industries) with $40 \mathrm{wt} \%$ tetraethylammonium hydroxide aqueous solution (SACEM Inc., Ultapure grade). Tetramethylammonium bimaleate, biphthalate and benzoate were, prepared by the neutralization of the acids with tetramethylammonium bicarbonate aqueous solution obtained from the reaction of trimethylamine with dimethyl carbonate in methanol ${ }^{20)}$. Asymmetric quaternary ammnonium bimaleates, biphthalates and benzoates were prepared similarly using asymmetric quaternary ammonium bicarbonate aqueous solutions obtained from the reaction of appropriate amines with dimethyl carbonate. Tetraethylammonium and tetrabutylammonium perchlorates (Tokyo Chemical Industry Co.) were 
recrystallized from high purity water and vacuum dried.

The exact concentrations of quaternary ammonium hydroxide and bicarbonate aqueous solutions were determined by potentiometric titration with a standard sulfuric acid aqueous solution using an auto titrator (Hiranuma Sangyo Co., COMTITE-8/UCB-7).

These salts were dissolved in $\gamma$-butyrolactone (Mitsubishi Petrochemical Co., F-GBL). The solutions were then vacuum dried until the water content dropped below $500 \mathrm{ppm}$, and were readjusted to desired concentrations by adding appropriate amount of the solvent.

The water contents in these stock solutions were measured by a moisture meter (Mitsubishi Kasei Co., CA-06). Their conductivities and densities at $25 \pm$ $0.1^{\circ} \mathrm{C}$ were measured by a conductivity meter (Toa Electronics, CM-30S) and a digital density meter (PAAR, DMA 45), respectively. Impurities in these stock solutions were determined by a X-ray fluorescence spectrometer (Rigaku Industrial Corp., 3370E) for $\mathrm{Cl}$ and an atomic absorption spectrometer (Hitachi, Z-9000) for $\mathrm{Na}, \mathrm{K}$ and Fe. These impurities were found less than $1 \mathrm{ppm}$.

Portions of each stock solution were placed in $100 \mathrm{ml}$ volumetric flasks and solutions for the conductivity measurements were prepared by successive weight dilutions. The conductivity measurements were carried out at $25 \pm 0.1^{\circ} \mathrm{C}$ by a bridge (Toa Electronics, CM25E) and a standard type conductivity cell with a cell constant $0.104 \mathrm{~cm}^{-1}$ (Toa Electronics, CG-2001PL).

The van der Waals" volumes of each ion were calculated from the molecular models constructed by a molecular modeling system (CHEMLAB-II, Molecular Design) on a computer (Digital Equipment Corp., VAX8350). Final optimization in the structure was performed by a MNDO molecular orbital calculation.

\section{RESULTS AND DISCUSSION}

The relevant properties of $\gamma$-butyrolactone used in this work are summarized in Table 1 , where $d, \eta, \varepsilon_{r}$ and $\kappa$ are density, viscosity, relative permittivity and electrolytic conductivity, respectively ${ }^{5)}$.

During the course of pursuing an electrolyte which exhibits the highest conductivity, the structural optimization of a cation in quaternary ammonium carboxylate $/ \gamma$-butyrolactone electrolytes has been carried out. Triethylmethylammonium salts have shown lower conductivities than tetraethylammonium counterparts at higher solute concentrations, particulaly for benzoate electrolytes as shown in Fig. 1, where electrolytic conductivities $\sigma$ of each electrolyte are plotted against solute concentration $C_{w}$. In order to elucidate this phenomenon, the conductometric analysis of these electrolytes has been attempted.

Table 1. Physical properties of $\gamma$-butyrolactone at $25^{\circ} \mathrm{C}$.

\begin{tabular}{lccc}
\hline$d / \mathrm{g} \mathrm{cm}^{-3}$ & $\eta / \mathrm{P}$ & $\varepsilon_{r} /-$ & $\kappa / \mathrm{S} \mathrm{cm}^{-1}$ \\
\hline 1.1234 & $1.727 \times 10^{-2}$ & 41.77 & $2 \times 10^{-8}$ \\
\hline
\end{tabular}

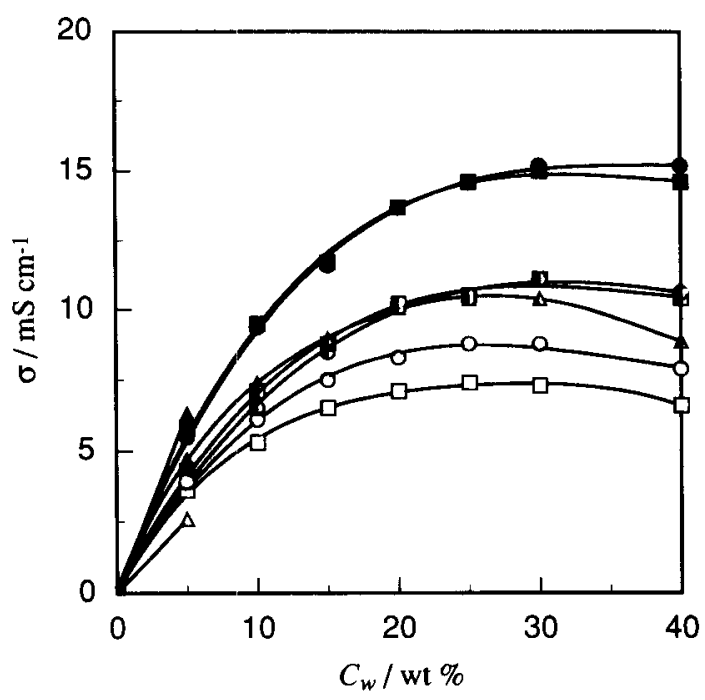

Fig. 1. Concentration dependence of the conductivities of quaternary ammonium carboxylate/ $\gamma$-butyrolactone electrolytes at $25^{\circ} \mathrm{C}$.
- $\mathrm{Et}_{4} \mathrm{~N}$ bimaleate
- $\mathrm{MeEt}_{3} \mathrm{~N}$ bimaleate
- $\mathrm{Me}_{4} \mathrm{~N}$ bimaleate
- $\mathrm{Et}_{4} \mathrm{~N}$ biphthalate
$\square \mathrm{MeEt}_{3} \mathrm{~N}$ biphthalate
$\triangle \mathrm{Me}_{4} \mathrm{~N}$ biphthalate
- $\mathrm{Et}_{4} \mathrm{~N}$ benzoate
ㅁ $\mathrm{MeEt}_{3} \mathrm{~N}$ benzoate
$\triangle \mathrm{Me}_{4} \mathrm{~N}$ benzoate 


\subsection{Molar conductivities}

The conductivities of the stock solutions of quaternary ammonium bimaleates, biphthalates and benzoates are given in Table 2. The concentrations of the stock solutions were adjusted to $1 \mathrm{~mol} \mathrm{dm}^{-3}$. The concentrations of tetramethylammonium bimaleate, tetramethylammonium benzoate and trimethylethylammonium benzoate were adjusted to $0.1 \mathrm{~mol} \mathrm{dm}^{-3}$ due to their low solubility in $\gamma$-butyrolactone as shown in Fig. 1.

These stock solutions were diluted successively and the molar concentrations $C$ on a volume basis were calculated from the concentration on a weight basis and the density of each solution. The densities were calculated from equation 1 , where $d$ and $d_{o}$ are the densities of solution and solvent, respectively, $m$ is the moles of solute per $\mathrm{kg}$ of solution, and $D$ is a constant characteristic of the electrolyte. Density data for the stock solutions given in Table 3 were used to obtain values of $D$.

$$
d=d_{0}+D m
$$

The molar conductivities $\Lambda$ were calculated from the experimental electrolytic conductivities $\sigma$ after correction for the electrolytic conductivity of the solvent $\kappa$. The molar conductivities over the concentration range of $10^{-2}$ to $10^{-3} \mathrm{~mol} \mathrm{dm}^{-3}$ are given in Tables 4, 5, 6 and 7 for quaternary ammonium perchlorate, bimaleate, biphthalate and benzoate electrolytes, respectively.

\subsection{Analysis of conductivity data}

The data were analyzed in terms of the Shedlovsky equation $^{21)}$

$$
\frac{1}{\Lambda S(z)}=\frac{1}{\Lambda_{0}}+\frac{C \Lambda S(z) f_{ \pm}^{2} K_{A}}{\Lambda_{0}{ }^{2}}
$$

where $S_{(2)}, f_{ \pm}, \Lambda_{0}$ and $K_{A}$ are the Shedlovsky function, a mean molar activity coefficient, a limiting molar conductivity and an association constant, respectively.

$$
\begin{aligned}
& S(z)=\left[z / 2+\sqrt{1+(z / 2)^{2}}\right]^{2} \\
& z=\frac{S \sqrt{C \Lambda}}{\Lambda_{0}^{3 / 2}}
\end{aligned}
$$

where $S$ is the Onsager coefficient

$$
\begin{aligned}
& S=\alpha \Lambda_{0}+\beta \\
& \alpha=\frac{8.204 \times 10^{5}}{\left(\varepsilon_{r} T\right)^{3 / 2}} \\
& \beta=\frac{82.49}{\eta\left(\varepsilon_{r} T\right)^{1 / 2}}
\end{aligned}
$$

\begin{tabular}{|c|c|c|c|}
\hline \multirow{2}{*}{$\frac{\sigma / \mathrm{mS} \mathrm{cm}^{-1}}{\mathrm{Me}_{4} \mathrm{~N}^{+}}$} & \multirow{2}{*}{$\frac{\left[\begin{array}{l}\mathrm{COO} \\
\mathrm{COOH}\end{array}\right.}{2.7^{*}}$} & \multirow{2}{*}{$\frac{I_{\mathrm{CO}}^{\mathrm{CO}}}{10.1}$} & \multirow{2}{*}{$\frac{7-C}{1.5^{*}}$} \\
\hline & & & \\
\hline \multirow[t]{2}{*}{$\mathrm{Me}_{3} \mathrm{EtN}^{+}$} & 12.9 & 10.5 & $1.6^{*}$ \\
\hline & 13.3 & 10.5 & 5.9 \\
\hline $\mathrm{Me}_{2} \mathrm{Et}_{2} \mathrm{~N}^{+}$ & 13.7 & 10.8 & 6.0 \\
\hline $\mathrm{MeEt}_{3} \mathrm{~N}^{+}$ & 13.7 & 10.8 & 6.8 \\
\hline $\mathrm{Et}_{4} \mathrm{~N}^{+}$ & 13.8 & 10.5 & 8.3 \\
\hline
\end{tabular}

Table 2. Conductivities of the $1 \mathrm{~mol} \mathrm{dm}^{-3}$ - quaternary ammonium carboxylate $/ \gamma$-butyrolactone electrolytes

\begin{tabular}{|c|c|c|c|}
\hline$d / \mathrm{g} \mathrm{cm}^{-3}$ & {$\left[\mathrm{COO}_{\mathrm{COH}}^{-}\right.$} & $-\mathrm{COO}^{-}$ & 1) $\mathrm{COO}$ \\
\hline $\mathrm{Me}_{4} \mathrm{~N}^{+}$ & $1.1241^{*}$ & 1.1404 & $1.1231^{*}$ \\
\hline $\mathrm{Me}_{3} \mathrm{EtN}^{+}$ & 1.1279 & 1.1379 & $1.1230^{*}$ \\
\hline$[\mathrm{N}+=\mathrm{Me}$ & 1.1362 & 1.1453 & 1.1260 \\
\hline $\mathrm{Me}_{2} \mathrm{Et}_{2} \mathrm{~N}^{+}$ & 1.1249 & 1.1346 & 1.1157 \\
\hline $\mathrm{MeEt}_{3} \mathbf{N}^{+}$ & 1.1227 & 1.1324 & 1.1149 \\
\hline $\mathrm{Et}_{4} \mathrm{~N}^{+}$ & 1.1200 & 1.1300 & 1.1128 \\
\hline
\end{tabular}
at $25^{\circ} \mathrm{C}$.

* $0.1 \mathrm{~mol} \mathrm{dm}^{-3}$

Table 3. Densities of the $1 \mathrm{~mol} \mathrm{dm}^{-3}$ - quaternary ammonium carboxylate $/ \gamma$-butyrolactone electrolytes at $25^{\circ} \mathrm{C}$.

* $0.1 \mathrm{~mol} \mathrm{dm}-3$ 
The mean molar activity coefficient $f_{+}$was calculated by the Debye-Hückel equation

$$
\begin{aligned}
& \log f_{ \pm}=-\frac{A \sqrt{\gamma C}}{1+B a \sqrt{\gamma C}} \\
& A=\frac{1.8246 \times 10^{6}}{\left(\varepsilon_{r} T\right)^{3 / 2}} \\
& B=\frac{50.29 \times 10^{8}}{\left(\varepsilon_{r} T\right)^{1 / 2}}
\end{aligned}
$$

where $a$ is the distance of closest approach in $\mathrm{cm}$ and $\gamma$ is the degree of dissociation in the mass action equation.

$$
K_{A}=\frac{1-\gamma}{C \gamma^{2} f_{ \pm}^{2}}
$$

where the activity coefficient for the neutral ion pair is assumed to equal unity.

The degree of dissociation $\gamma$ is correlated with the Shedlovsky function by the following equation.

$$
\gamma=\frac{\Lambda}{\Lambda_{0}} S(z)
$$

The ion-size parameter $a$ in the equation 8 was estimated by summing up ionic radii $r$ 's of a cation and an anion, which were calculated by equation 13 using the van der Waals' volume $V$ of each ion. The ionic radii of quaternary ammonium ions and carboxylate ions are given in Table 8 . The estimated values for quaternary ammoium ions are very close to those obtained by a similar method ${ }^{22)}$. The ionic radius of perchlorate ion was assumed to be $2.00 \AA^{4)}$.

$$
r=\left(\frac{3 V}{4 \pi}\right)^{1 / 3}
$$

A set of parameters $\Lambda_{0}$ and $K_{\mathrm{A}}$ are derived from the intercept and slope of the straight line on the plot of

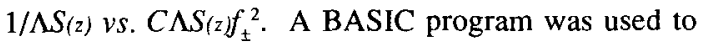
solve the above procedures, where an initial value of $\Lambda_{0}$ was calculated from the Arrhenius-Ostwald relation $\left(S(z)=f_{ \pm}=1\right.$ in equation 2 ) and iterations were continued until the error in $\Lambda_{0}$ became less than 0.001 . A typical result is given in Table 9. Derived parameters are summarized in Tables 10, 11 and 12.
Table 4. Molar concentrations and molar conductivities of quaternary ammonium perchlorate/

\begin{tabular}{|c|c|c|c|}
\hline $10^{3} \mathrm{C}$ & $\Lambda$ & $10^{3} \mathrm{C}$ & $\Lambda$ \\
\hline \multicolumn{2}{|c|}{$\mathrm{Me}_{4} \mathrm{~N}$ bimaleate } & \multicolumn{2}{|c|}{$\mathrm{Me}_{3} \mathrm{EtN}$ bimaleate } \\
\hline 9.7352 & 40.213 & 9.7407 & 39.780 \\
\hline 7.7479 & 40.989 & 8.2007 & 40.433 \\
\hline 5.9551 & 41.960 & 6.3841 & 41.272 \\
\hline 3.6390 & 43.220 & 4.3085 & 42.400 \\
\hline 1.9542 & 44.587 & 1.9471 & 44.046 \\
\hline 1.0916 & 45.647 & 0.9751 & 45.174 \\
\hline \multicolumn{2}{|c|}{$\left[\mathrm{N}-\mathrm{Me}_{\mathrm{Me}}\right.$ bimaleate } & \multicolumn{2}{|c|}{$\mathrm{Me}_{2} \mathrm{Et}_{2} \mathrm{~N}$ bimaleate } \\
\hline 9.9679 & 39.485 & 9.9887 & 38.972 \\
\hline 8.3737 & 40.087 & 7.9113 & 39.903 \\
\hline 6.5422 & 40.946 & 5.8964 & 40.734 \\
\hline 4.5274 & 41.985 & 3.9468 & 41.826 \\
\hline 2.5150 & 43.212 & 1.9774 & 42.990 \\
\hline 1.2196 & 44.488 & 1.0196 & 44.147 \\
\hline \multicolumn{2}{|c|}{$\mathrm{MeEt}_{3} \mathrm{~N}$ bimaleate } & \multicolumn{2}{|c|}{$\mathrm{Et}_{4} \mathrm{~N}$ bimaleate } \\
\hline 9.9253 & 38.839 & 9.8916 & 38.779 \\
\hline 7.9012 & 39.512 & 7.7222 & 39.507 \\
\hline 6.0870 & 40.199 & 5.8570 & 40.171 \\
\hline 4.1749 & 41.148 & 3.7297 & 41.177 \\
\hline 2.0363 & 42.576 & 2.0008 & 42.324 \\
\hline 1.0099 & 43.628 & 1.0265 & 43.235 \\
\hline
\end{tabular}
$\gamma$-butyrolactone electrolytes at $25^{\circ} \mathrm{C}$.

\begin{tabular}{llll}
\hline $\begin{array}{l}10^{3} \mathrm{C} \\
/ \mathrm{mol} \mathrm{dm}^{-3} / \mathrm{S} \mathrm{cm}^{2} \mathrm{~mol}^{-1}\end{array}$ & $10^{3} \mathrm{C}$ & \multicolumn{3}{c}{} \\
\hline $\mathrm{Et}_{4} \mathrm{~N} \mathrm{ClO}_{4}$ & \multicolumn{3}{c}{$\mathrm{Bu}_{4} \mathrm{~N} \mathrm{ClO}_{4}$} \\
9.7352 & 40.213 & 9.7407 & 39.780 \\
7.7479 & 40.989 & 8.2007 & 40.433 \\
5.9551 & 41.960 & 6.3841 & 41.272 \\
3.6390 & 43.220 & 4.3085 & 42.400 \\
1.9542 & 44.587 & 1.9471 & 44.046 \\
1.0916 & 45.647 & 0.9751 & 45.174 \\
\hline
\end{tabular}

Table 5. Molar concentrations and molar conductivities of quaternary ammonium bimaleate/ $\gamma$-butyrolactone electrolytes at $25^{\circ} \mathrm{C}$. 
Table 6. Molar concentrations and molar conductivities of quaternary ammonium biphthalate/ $\gamma$-butyrolactone electrolytes at $25^{\circ} \mathrm{C}$.

\begin{tabular}{|c|c|c|c|}
\hline $10^{3} \mathrm{C}$ & $\Lambda$ & $10^{3} \mathrm{C}$ & $\Lambda$ \\
\hline \multicolumn{2}{|c|}{$\mathrm{Me}_{4} \mathrm{~N}$ biphthalate } & \multicolumn{2}{|c|}{$\mathrm{Me}_{3} \mathrm{EtN}$ biphthalate } \\
\hline 9.9677 & 35.536 & 10.0582 & 36.038 \\
\hline 7.9319 & 36.647 & 8.0444 & 36.781 \\
\hline 6.1130 & 37.360 & 6.0054 & 37.546 \\
\hline 4.1578 & 38.405 & 4.0345 & 38.832 \\
\hline 2.1405 & 39.976 & 2.0391 & 39.885 \\
\hline 1.1319 & 40.859 & 1.0332 & 40.972 \\
\hline \multicolumn{2}{|c|}{$\mathrm{C}_{\mathrm{Me}}^{\mathrm{Me}}$ biphthalate } & \multicolumn{2}{|c|}{$\mathrm{Me}_{2} \mathrm{Et}_{2} \mathrm{~N}$ biphthalate } \\
\hline 9.7567 & 35.963 & 9.8348 & 35.606 \\
\hline 7.9368 & 36.624 & 7.9977 & 36.233 \\
\hline 6.0204 & 37.386 & 6.1726 & 37.032 \\
\hline 4.0393 & 38.467 & 4.224 .1 & 37.992 \\
\hline 1.9767 & 39.723 & 2.0479 & 39.299 \\
\hline 1.0320 & 40.599 & 1.0576 & 40.215 \\
\hline \multicolumn{2}{|c|}{$\mathrm{MeEt}_{3} \mathrm{~N}$ biphthalate } & \multicolumn{2}{|c|}{$\mathrm{Et}_{4} \mathrm{~N}$ biphthalate } \\
\hline 9.9849 & 35.136 & 9.9316 & 34.514 \\
\hline 8.3456 & 35.561 & 8.1159 & 35.077 \\
\hline 6.4731 & 36.193 & 5.9566 & 35.923 \\
\hline 4.4558 & 37.071 & 4.0016 & 36.680 \\
\hline 2.4753 & 38.239 & 2.6788 & 37.304 \\
\hline 1.2917 & 39.327 & 1.4889 & 38.237 \\
\hline
\end{tabular}

Table 7. Molar concentrations and molar conductivities of quaternary ammonium benzoate $/ \gamma$ butyrolactone electrolytes at $25^{\circ} \mathrm{C}$.

\begin{tabular}{|c|c|c|c|}
\hline $10^{3} \mathrm{C}$ & $\Lambda$ & $10^{3} \mathrm{C}$ & $\Lambda$ \\
\hline \multicolumn{2}{|c|}{$\mathrm{Me}_{4} \mathrm{~N}$ benzoate } & \multicolumn{2}{|c|}{$\mathrm{Me}_{3} \mathrm{EtN}$ benzoate } \\
\hline 9.9975 & 29.505 & 9.9984 & 30.483 \\
\hline 8.3015 & 30.570 & 7.9466 & 31.898 \\
\hline 6.1986 & 32.230 & 5.9711 & 33.324 \\
\hline 4.3194 & 34.097 & 4.1141 & 34.875 \\
\hline 2.0599 & 36.527 & 2.0683 & 36.919 \\
\hline 1.0337 & 37.943 & 1.0424 & 37.903 \\
\hline \multicolumn{2}{|c|}{$\mathrm{C}_{\mathrm{N}}=\mathrm{Me}_{\mathrm{Me}}^{\mathrm{Me}}$ benzoate } & \multicolumn{2}{|c|}{$\mathrm{Me}_{2} \mathrm{Et}_{2} \mathrm{~N}$ benzoate } \\
\hline 10.0056 & 31.191 & 10.0892 & 31.705 \\
\hline 8.0117 & 32.350 & 7.9887 & 32.744 \\
\hline 5.9877 & 33.666 & 6.0493 & 33.868 \\
\hline 4.0557 & 35.205 & 4.1092 & 35.233 \\
\hline 1.9935 & 37.111 & 2.0969 & 37.046 \\
\hline 1.0259 & 37.840 & 1.1204 & 37.881 \\
\hline \multicolumn{2}{|c|}{$\mathrm{MeEt}_{3} \mathrm{~N}$ benzoate } & \multicolumn{2}{|c|}{$\mathrm{Et}_{4} \mathrm{~N}$ benzoate } \\
\hline 9.9857 & 32.424 & 10.1772 & 32.689 \\
\hline 7.9811 & 33.351 & 8.2609 & 33.372 \\
\hline 6.1242 & 34.319 & 6.3718 & 34.084 \\
\hline 4.1484 & 35.503 & 4.4023 & 34.954 \\
\hline 2.0322 & 36.905 & 2.4429 & 36.117 \\
\hline 1.0127 & 37.682 & 1.2805 & 36.971 \\
\hline
\end{tabular}

Table 8. Estimated ionic radii from van der Waals' volumes.

\begin{tabular}{lllll}
\hline Cation & $r_{+} / \AA$ & & Anion & $r_{.} / \AA$ \\
\hline $\mathrm{Me}_{4} \mathrm{~N}^{+}$ & 2.89 & $2.83^{* 1}$ & $\mathrm{COO}_{\mathrm{COH}}^{-}$ & 2.64 \\
$\mathrm{Me}_{3} \mathrm{EtN}^{+}$ & 3.05 & & \\
$\mathrm{~N}^{+} \mathrm{Me}$ & 3.08 & & & \\
$\mathrm{Me}_{2} \mathrm{Et}_{2} \mathrm{~N}^{+}$ & 3.19 & & \\
$\mathrm{MeEt}_{3} \mathrm{~N}^{+}$ & 3.32 & & \\
$\mathrm{Et}_{4} \mathrm{~N}^{+}$ & 3.42 & $3.39^{* 1}$ & & \\
$\mathrm{Bu}_{4} \mathrm{~N}^{+}$ & 4.20 & $4.15^{* 1}$ & & \\
\hline
\end{tabular}

*1 Data from literature 22$)$ 
Table 9. The calculated result for tetramethylammonium benzoate/ $\gamma$-butyrolactone electrolyte.

\begin{tabular}{llllllllll}
\hline $103 C$ & $\Lambda$ & $z$ & $S_{(z)}$ & $\gamma$ & $f_{ \pm}$ & $C \Lambda S_{(z)} f_{ \pm}{ }^{2}$ & $1 / \Lambda S_{(z)}$ & $\Lambda_{0}$ & $K_{A}$ \\
\hline 9.9975 & 29.505 & 0.1361 & 1.1457 & 0.8113 & 0.8017 & 0.2172 & 0.0296 & 39.805 & 46.978 \\
8.3015 & 30.570 & 0.1262 & 1.1345 & 0.8324 & 0.8131 & 0.1903 & 0.0288 & 41.738 & 42.282 \\
6.1986 & 32.230 & 0.1120 & 1.1185 & 0.8652 & 0.8301 & 0.1540 & 0.0277 & 41.661 & 43.457 \\
4.3194 & 34.097 & 0.0962 & 1.1009 & 0.9010 & 0.8500 & 0.1172 & 0.0266 & 41.664 & 43.413 \\
2.0599 & 36.527 & 0.0687 & 1.0711 & 0.9391 & 0.8874 & 0.0635 & 0.0256 & 41.66443 .415 \\
1.0337 & 37.943 & 0.0496 & 1.0509 & 0.9570 & 0.9158 & 0.0346 & 0.0251 & $(a=5.72 \AA)$ \\
\hline
\end{tabular}

Table 10. Derived limiting molar conductivities.

\begin{tabular}{|c|c|c|c|}
\hline $\begin{array}{l}\Lambda_{0} \\
/ \mathrm{S} \mathrm{cm}^{2} \mathrm{~mol}^{-1}\end{array}$ & {$\left[\begin{array}{l}\mathrm{COO}^{-} \\
\mathrm{COOH}\end{array}\right.$} & $\pi_{\mathrm{COOH}}^{\mathrm{COO}}$ & $\Rightarrow \mathrm{COO}$ \\
\hline $\mathrm{Me}_{4} \mathrm{~N}^{+}$ & 48.14 & 43.48 & 41.66 \\
\hline $\mathrm{Me}_{3} \mathrm{EtN}^{+}$ & 47.53 & 43.27 & 41.37 \\
\hline$\left[\begin{array}{l}\mathrm{Ne} \\
\mathrm{Me}\end{array}\right.$ & 47.10 & 42.90 & 41.03 \\
\hline $\mathrm{Me}_{2} \mathrm{Et}_{2} \mathrm{~N}^{+}$ & 46.45 & 42.53 & 40.89 \\
\hline $\mathrm{MeEt}_{3} \mathrm{~N}^{+}$ & 45.84 & 41.67 & 40.27 \\
\hline $\mathrm{Et}_{4} \mathrm{~N}^{+}$ & 45.43 & 40.75 & 39.46 \\
\hline
\end{tabular}

Table 11. Derived ion association constants.

\begin{tabular}{|c|c|c|c|}
\hline $\begin{array}{l}K_{A} \\
/ \mathrm{mol}^{-1} \mathrm{dm}^{3}\end{array}$ & {$\left[\begin{array}{l}\mathrm{L}_{\mathrm{COOH}} \\
\mathrm{COO}^{-}\end{array}\right.$} & $\pi_{\mathrm{CO}}^{\mathrm{CO}}$ & $\Rightarrow \mathrm{COO}$ \\
\hline $\mathrm{Me}_{4} \mathrm{~N}^{+}$ & 8.36 & 9.64 & 43.42 \\
\hline $\mathrm{Me}_{3} \mathrm{EtN}^{+}$ & 7.48 & 6.45 & 31.34 \\
\hline $\mathrm{N}^{\circ}-\mathrm{Me}$ & 6.68 & 5.55 & 24.04 \\
\hline $\mathrm{Me}_{2} \mathrm{Et}_{2} \mathrm{~N}^{+}$ & 5.94 & 5.50 & 19.84 \\
\hline $\mathrm{MeEt}_{3} \mathrm{~N}^{+}$ & 4.59 & 4.13 & 11.24 \\
\hline $\mathrm{Et}_{4} \mathrm{~N}^{+}$ & 3.00 & 2.42 & 5.51 \\
\hline
\end{tabular}

Table 12. Derived parameters for perchlorates.

\begin{tabular}{lcc}
\hline & $\Lambda_{0}$ & $K_{A}$ \\
\hline $\mathrm{Et}_{4} \mathrm{~N} \mathrm{ClO}_{4}$ & 49.98 & 2.66 \\
$\mathrm{Bu}_{4} \mathrm{~N} \mathrm{ClO}_{4}$ & 43.81 & 1.75 \\
\hline
\end{tabular}

\subsection{Single ion limiting molar conductivities}

Conductivity data of $\mathrm{Et}_{4} \mathrm{NClO}_{4}$ and $\mathrm{Bu}_{4} \mathrm{NClO}_{4}$ in $\gamma$ butyrolactone were also analyzed in order to generate single ion limiting molar conductivities from Table 10, because the single ion limiting molar conductivity of $\mathrm{Bu}_{4} \mathrm{~N}^{+}$is known ${ }^{4}$. However, this reported value seems to be too small, because the transport number $t_{0}^{+}$ $\left(\mathrm{Bu}_{4} \mathrm{NClO}_{4}\right)$ and the relative difference between $\lambda_{\mathrm{o}}{ }^{+}\left(\mathrm{Et}_{4} \mathrm{~N}^{+}\right)$and $\lambda_{\mathrm{o}}{ }^{+}\left(\mathrm{Bu}_{4} \mathrm{~N}^{+}\right)$designated as $\Delta$ are deviated from those obtained for other dipolar aprotic solvents as shown in Table 13. Assuming $t_{0}^{+}\left(\mathrm{Bu}_{4} \mathrm{NClO}_{4}\right)$ is a more consistent value 0.329 , which is calculated from the other reported data ${ }^{23)}, \lambda_{\mathrm{o}}{ }^{+}\left(\mathrm{Bu}_{4} \mathrm{~N}^{+}\right)$was reassigned. As the result, $\lambda_{0}{ }^{+}\left(\mathrm{Et}_{4} \mathrm{~N}^{+}\right)$was proven to be 20.6 , which is almost the same as the reported value ${ }^{4}$.

Table 13. Mobility data of ions in dipolar aprotic solvents at $25^{\circ} \mathrm{C}$.

\begin{tabular}{|c|c|c|c|c|c|}
\hline & $\mathrm{GBL}^{* 1}$ & $\mathrm{GBL}^{* 2} \mathrm{D}$ & $\mathrm{MF}^{* 3} \mathrm{D}$ & $\mathrm{MSO}^{* 4}$ & $\mathrm{PC}^{* 5}$ \\
\hline$\lambda_{0}+\left(\mathrm{Bu}_{4} \mathrm{~N}^{+}\right)$ & 14.4 & 11.8 & 25.4 & 11.8 & 9.0 \\
\hline$\lambda_{\mathrm{o}}+\left(\mathrm{Et}_{4} \mathrm{~N}^{+}\right)$ & 20.6 & 20.4 & 35.4 & 17.5 & 13.2 \\
\hline$\lambda_{\mathrm{o}}-\left(\mathrm{ClO}_{4}^{-}\right)$ & 29.4 & 26.2 & 52.4 & 24.1 & 18.4 \\
\hline$t_{\mathrm{o}}+\left(\mathrm{Bu}_{4} \mathrm{NClO}_{4}\right.$ & 0.329 & 0.311 & 0.326 & 0.329 & 0.328 \\
\hline$\Delta^{* 6}$ & 0.142 & 0.226 & 0.129 & 0.159 & 0.153 \\
\hline \multicolumn{6}{|c|}{ 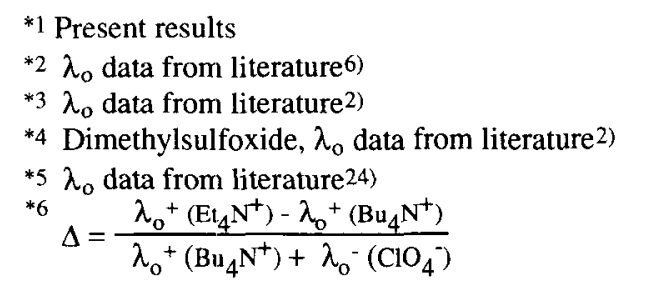 } \\
\hline
\end{tabular}


The resulting consistent set of the single ion limiting molar conductivities of quaternary ammonium ions and carboxylate ions are given in Table 14.

Walden products $\lambda_{0} \eta$ of each ion are plotted against the reciprocal of ionic radius $r$ in Fig. 2. The theoretical behavior calculated from Stokes' law in equation 14 is given by the bottom line for perfect stick $\left(A_{s}=0.82\right)$ and by the top line for perfect $\operatorname{slip}\left(A_{s}=1.23\right)$.

$$
\lambda_{0} \eta=\frac{A_{s}}{r}
$$

Table 14. Single ion molar conductivities in $\gamma$ butyrolactone at $25^{\circ} \mathrm{C}$.

\begin{tabular}{|c|c|c|c|}
\hline \multicolumn{2}{|c|}{ 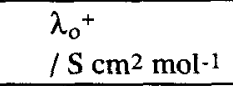 } & \multicolumn{2}{|c|}{$\begin{array}{l}\lambda_{0}- \\
/ S \mathrm{~cm}^{2} \mathrm{~mol}^{-1} \\
\end{array}$} \\
\hline $\mathrm{Me}_{4} \mathrm{~N}^{+}$ & 23.1 & ${ }^{\mathrm{COO}_{\mathrm{COOH}}^{-}}$ & 24.8 \\
\hline $\mathrm{Me}_{3} \mathrm{EtN}^{+}$ & 22.7 & & 20.2 \\
\hline $\mathrm{N}+\mathrm{Me}$ & 22.3 & $-\mathrm{C}$ & 18.9 \\
\hline $\mathrm{Me}_{2} \mathrm{Et}_{2} \mathrm{~N}^{+}$ & 22.0 & & \\
\hline $\mathrm{MeEt}_{3} \mathbf{N}^{+}$ & 21.3 & & \\
\hline $\mathrm{Et}_{4} \mathrm{~N}^{+}$ & 20.6 & & \\
\hline
\end{tabular}

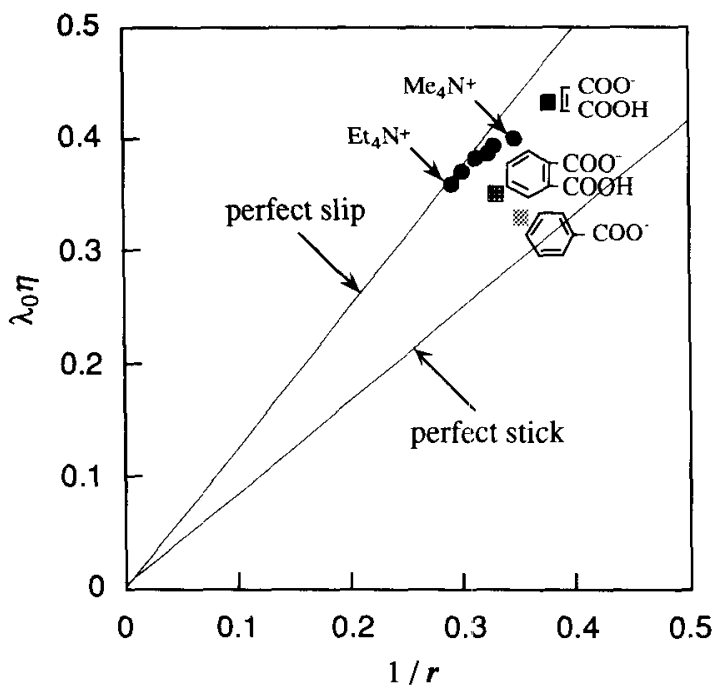

Fig. 2. Walden products as a function of the reciprocal of ionic radius.
All quaternary ammonium ions including tetramethylammonium ion follow the theoretical behavior for perfect slip. Bimaleate, biphthalate and benzoate ions also approach the behavior for perfect slip. This result suggests that all ions discussed here are able to move without adhesion to $\gamma$-butyrolactone. The inversion of the order in the mobility between biphthalate and benzoate ion, which cannot be seen in the aqueous solution ${ }^{25}$, may be explained by the lower conductivity of benzoate solution over the experimental concentration range due to the ion association discussed later.

\subsection{Association constants}

Derived ion association constants in Table 11 are plotted against ionic radii of cations as shown in Fig. 3. The $K_{A}$ values increase in the order, $\mathrm{Et}_{4} \mathrm{~N}^{+}<\mathrm{MeEt}_{3} \mathrm{~N}^{+}<$ $\mathrm{Me}_{2} \mathrm{Et}_{2} \mathrm{~N}^{+}<\mathrm{Me}_{2}\left(\mathrm{CH}_{2}\right)_{4} \mathrm{~N}^{+}<\mathrm{Me}_{3} \mathrm{EtN}^{+}<\mathrm{Me}_{4} \mathrm{~N}^{+}$with decreasing cation size. This tendency can be predicted theoretically by the Bjerrum equation

$$
\begin{aligned}
& K_{B}=\frac{3.531 \times 10^{13}}{\left(\varepsilon_{r} T\right)^{3}} \int_{2}^{b} \chi^{-4} \exp (\chi) \mathrm{d} \chi(15) \\
& b=\frac{1.671 \times 10^{-3}}{a \varepsilon_{r} T}
\end{aligned}
$$

where the ion association constant $K_{B}$ increases as the ion-size parameter $a$ decreases.

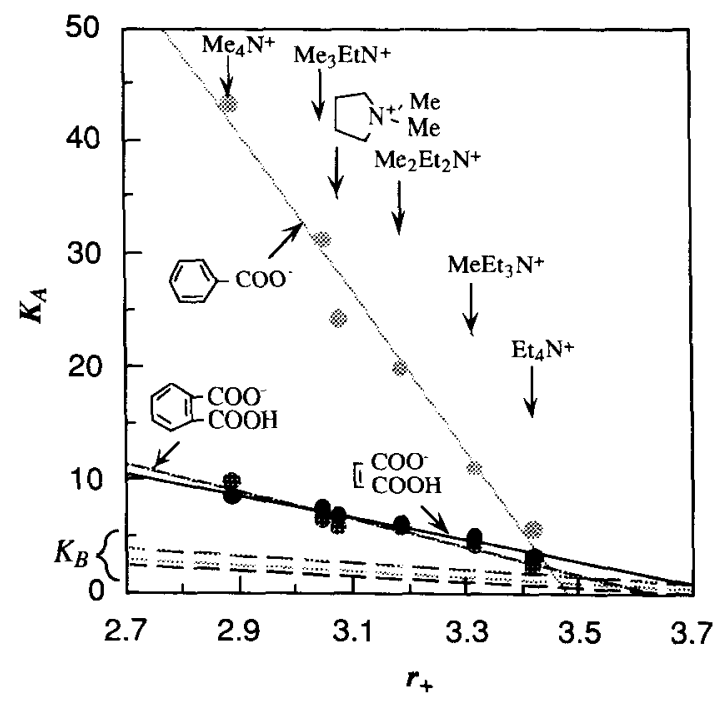

Fig. 3. Effect of cation size on ion association constants. 
Calculated $K_{B}$ values are shown by the broken curves in Fig. 3. The fact that the $K_{B}$ values are smaller than the experimental $K_{A}$ values indicates the existence of iondipole and dipole-dipole interaction besides an electrostatic force due to the delocalizability of the carboxylate ions.

A large dependence of cation size on the ion association constant observed for benzoate solutions cannot be explained theoretically. However this result is closely related to the low ionization constant $K a$ of benzoic acid in $\gamma$-butyrolactone compared with maleic acid and phthalic acid.

The ionization constants of carboxylic acids are given in Table $15^{26)}$, where the values in $\gamma$-butyrolactone are estimated by the data that $p K a$ values in $\gamma$-butyrolactone are smaller than those in propylene carbonate by 2 units $^{27)}$.

\section{RFFERENCES}

1) R. A. Robinson and R. H. Stokes, Electrolyte Solutions, 2nd ed. rev., Butterworths (1959).

2) A. K. Convington and T. Dickinson, ed., Physical Chemistry of Organic Solvent Systems, Plenum Press, London (1973).

3) O. Popovych and R. P. T. Tomkins, Nonaqueous Solution Chemistry, John Wiley \& Sons, New York (1981).

4) M. Salomon and E. J. Plichta, Electrochim. Acta, 29, 731 (1984).

5) M. Salomon and E. J. Plichta, Electrochim. Acta, 30, 113 (1985).

6) L. Werblan, A. Balkowska, J. Lensinski, and G. Szymanski, J. Electroanal. Chem., 250, 165 (1988).

7) A. Balkowska, G. Szymanski and L. Werblan, J. Electroanal. Chem., 287, 229 (1990).

8) A. Balkowska, J. Lensinski, G. Szymanski and L. Werblan, J. Electroanal. Chem., 289, 171 (1990).

9) M. Ue, T. Sato, K. Ida and S. Mori, lst West Pacific Electrochemistry Symposium, 1A-11 (1992).

10) M. Ue, T. Sato, H. Asahina, K. Ida and S. Mori, Denki Kagaku, 60, 480 (1992).

11) S. Mori and M. Ue, USP 4715976 (1987); JP 916646, 8092 (1991); EP 227433B (1992).

12) I. M. Kolthoff and M. K. Chantooni, Jr., J. Amer. Chem. Soc., 85, 426 (1963).

13) I. M. Kolthoff and M. K. Chantooni, Jr., J. Phys. Chem., 70, 856 (1966).
Lower conductivities of trietylmethylammonium salts than tetraethylammonium counterparts at higher concentrations (Fig. 1) can be predicted by the tendency of $K_{A}$ values obtained at low concentrations.

Table 15. Ionization constants of carboxylic acids in dipolar aprotic solvents at $25^{\circ} \mathrm{C}$.

\begin{tabular}{ccrcc}
\hline$p K a_{1}$ & $\mathrm{GBL}^{* 1}$ & $\mathrm{DMA}^{* 2}$ & $\mathrm{AN}$ & $\mathrm{PC}$ \\
\hline maleic acid & 10 & \multicolumn{1}{c}{4} & - & - \\
phthalic acid & 11 & 5 & 14 & - \\
salicylic acid & 14 & 7 & 17 & 15 \\
benzoic acid & 18 & 11 & 21 & 20 \\
\hline
\end{tabular}

${ }^{* 1}$ Estimated values

*2 Dimethylacetamide

14) I. M. Kolthoff and M. K. Chantooni, Jr., J. Amer. Chem. Soc., 97, 1376 (1975).

15) I. M. Kolthoff, M. K. Chantooni, Jr. and H. Smagowski, Anal. Chem., 42, 1622 (1970).

16) E. Roletto, A. Vanni and V. Zelano, Ann. Chimica, 70, 147 (1980).

17) E. Roletto, A. Vanni and V. Zelano, Ann. Chimica, 70, 375 (1980).

18) Z. Pawlak, R. A. Robinson and R. G. Bates, $J$. Solution Chem., 7, 631 (1978).

19) M. Ue, T. Sato and M. Takeda, 1992 Fall Meeting of the Electrochemical Society of Japan, Abstr. 2H14 (1992).

20) S. Mori, K. Ida and M. Ue, USP 4892944 (1990).

21) R. M. Fuoss and T. Shedlovsky, J. Amer. Chem. Soc., 71, 1496 (1949).

22) J. T. Edward, J. Chem. Educ., 47, 261 (1970).

23) A. D'Aprano, D. I. Donato and A. Carrubba, $J$. Solution Chem., 12, 209 (1983).

24) M. L. Jansen and H. L. Yeager, J. Phys. Chem., 77, 3089 (1973).

25) Unpublished results.

26) K. Izutsu, Acid-Base Dissociation Constants in Dipolar Aprotic Solvents, IUPAC Chemical Data Series No. 35, Blackwell Scientific, Oxford (1990).

27) K. Izutsu, T. Nakamura and H. Suzuki, 41 st Annual Meeting of the Japan Society for Analytical Chemistry, Abstr. 3H12 (1992). 